\title{
Comportement alimentaire et mérycique d'ovins et de bovins recevant des fourrages verts
}

\author{
J.P. DULPHY et Brigitte MICHALET-DOREAU \\ avec la collaboration technique de Madeleine Dudilieu, Jacqueline JAMot, \\ J.M. Boissau, H. Bousquet et L. L'Hotelier \\ I.N.R.A., C.R.Z.V. de Theix, Laboratoire des Aliments, F 63122 Ceyrat
}

\section{Résumé}

Nous avons distribué, simultanément à des moutons et des génisses de 1 an, 11 fourrages verts pour comparer les paramètres du comportement alimentaire et mérycique de ces animaux. Les génisses ont ingéré plus et plus longtemps que les moutons. Elles ont ruminé plus rapidement une même quantité de matière sèche. Les moutons et les génisses ont ingéré les mêmes quantités, à la même vitesse, durant les grands repas. Par contre, les génisses ont effectué plus de petits repas que les moutons et surtout ont ingéré plus durant ces petits repas.

Les différents paramètres du comportement alimentaire et mérycique des moutons et ceux des génisses ont été étroitement liés, à l'exception de la durée journalière d'ingestion et du nombre quotidien de repas. Lorsque la qualité du fourrage a augmenté, la durée d'ingestion a peu varié, mais la durée de rumination a diminué. Dans les mêmes conditions alors que les quantités ingérées par jour augmentaient, celles par grands repas ne variaient pas.

Nous avons enfin examiné par espèce animale les relations entre les paramètres du comportement et les quantités ingérées.

\section{Introduction}

Dans une série d'études précédentes (Dulphy \& Demarquilly, 1974; DulPhy \& BECHET, 1976), nous avons analysé les paramètres du comportement alimentaire et mérycique de moutons recevant des fourrages verts. Nous avions montré alors qu'il existait des liaisons parfois étroites entre ces paramètres et la composition des fourrages (cf. aussi Dulphy, Remond \& Theriez, 1979). Par la suite, dans le cadre d'études faites sur l'ingestibilité des ensilages d'herbe, nous avons enregistré, simultanément, le comportement de moutons et de génisses recevant les fourrages verts témoins. Il nous a paru intéressant de rassembler les résultats correspondants, les données bibliographiques sur ce sujet étant pratiquement inexistantes. 


\section{Matériel et méthodes}

Nous avons utilisé 11 fourrages verts. 8 ont été étudiés dans le cadre de nos essais sur les ensilages (Dulphy, Michalet-Doreau \& Demarquilly, 1984) et les 3 autres indépendamment de ces essais. La composition chimique moyenne de ces fourrages, au cours des périodes de mesure, est donnée dans le tableau 1.

\section{Tableau 1}

Composition chimique et digestibilité des fourrages étudiés.

Chemical composition and digestibility of studied forages.

\begin{tabular}{|c|c|c|c|c|c|c|}
\hline \multirow[b]{2}{*}{$\begin{array}{c}\text { Année } \\
\text { Year }\end{array}$} & \multirow[b]{2}{*}{$\begin{array}{l}\text { Espèce végétale } \\
\text { Forage species }\end{array}$} & \multirow{2}{*}{$\begin{array}{c}\text { Teneur } \\
\text { en } \\
\text { matière } \\
\text { sèche } \\
\text { Dry } \\
\text { matter } \\
\text { content }\end{array}$} & \multicolumn{3}{|c|}{$\begin{array}{l}\text { Teneur en \% de la MS } \\
\text { Composition in \% DM }\end{array}$} & \multirow{2}{*}{$\begin{array}{c}\text { Digestibilité } \\
\text { de la matière } \\
\text { organique } \\
\text { (moutons) } \\
\text { Organic } \\
\text { matter } \\
\text { digestibility } \\
\text { (sheep) }\end{array}$} \\
\hline & & & $\begin{array}{l}\text { Cendres } \\
\text { Ashes }\end{array}$ & $\begin{array}{c}\text { Matières } \\
\text { azotées } \\
\text { totales } \\
\text { Crude } \\
\text { protein }\end{array}$ & $\begin{array}{c}\text { Cellulose } \\
\text { brute } \\
\text { Crude } \\
\text { protein }\end{array}$ & \\
\hline 1972 & $\begin{array}{l}\text { Ray-grass anglais } \ldots . . \\
\text { Perennial rye-grass }\end{array}$ & 19,3 & 7,9 & 9,7 & 20,7 & 77,9 \\
\hline \multirow{2}{*}{1973} & $\begin{array}{l}\text { Ray-grass italien } \\
\text { Italian rye-grass }\end{array}$ & 16,9 & 11,2 & 13,4 & 26,3 & 65,5 \\
\hline & $\begin{array}{l}\text { Fétuque élevée } . . . \\
\text { Tall fescue }\end{array}$ & 16,2 & 11,4 & 12,3 & 32,7 & 61,9 \\
\hline \multirow{2}{*}{1974} & $\begin{array}{l}\text { Ray-grass italien } \\
\text { Italian rye-grass }\end{array}$ & 14,3 & 12,5 & 16,3 & 20,0 & 81,3 \\
\hline & $\begin{array}{l}\text { Luzerne } \\
\text { Lucerne }\end{array}$ & 22,8 & 11,0 & 18,5 & 27,5 & 62,8 \\
\hline \multirow{2}{*}{1975} & $\begin{array}{l}\text { Ray-grass italien } \ldots \ldots \\
\text { Italian rye-grass }\end{array}$ & 14,0 & 12,9 & 13,9 & 20,8 & 74,4 \\
\hline & $\begin{array}{l}\text { Fétuque élevée } . . . \\
\text { Tall fescue }\end{array}$ & 18,8 & 12,4 & 14,1 & 28,5 & 66,1 \\
\hline \multirow{4}{*}{1976} & $\begin{array}{l}\text { Ray-grass anglais } \ldots \\
\text { Perennial rye-grass }\end{array}$ & 17,7 & 10,1 & 16,7 & 19,3 & 75,2 \\
\hline & $\begin{array}{l}\text { Ray-grass anglais } \ldots \\
\text { Perennial rye-grass }\end{array}$ & 18,7 & 9,2 & 13,6 & 21,9 & 71,1 \\
\hline & $\begin{array}{l}\text { Dactyle } \ldots . . . \cdots \\
\text { Cocksfoot }\end{array}$ & 28,8 & 10,0 & 9,0 & 31,5 & 63,3 \\
\hline & $\begin{array}{l}\text { Ray-grass italien } \ldots \\
\text { Italian rye-grass }\end{array}$ & 27,6 & 9,8 & 14,0 & 25,2 & 64,0 \\
\hline
\end{tabular}


TABleau 2

Valeurs movennes des différents paramètres du comportement (II résultats pour chaque valeur moyenne).

Mean values of the behavioural parameters (11 results for each mean value).

\begin{tabular}{|c|c|c|c|}
\hline & $\begin{array}{l}\text { Moutors } \\
\text { Sheep }\end{array}$ & $\begin{array}{l}\text { Génisses } \\
\text { Heifers }\end{array}$ & $\begin{array}{l}\text { Signification } \\
\text { de la différence } \\
\text { Significance } \\
\text { of the difference }\end{array}$ \\
\hline \multicolumn{4}{|l|}{ Journée - Day } \\
\hline 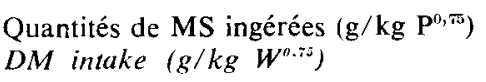 & $68,4 \pm 11,8$ & $89.7 \pm 13,1$ & $\mathrm{P}<0,05$ \\
\hline $\begin{array}{l}\text { Durée journalière d'ingestion (mn) } \\
\text { Daily time spent eating }\end{array}$ & $314 \pm 29$ & $410 \pm 36$ & $\mathrm{P}<0,05$ \\
\hline $\begin{array}{l}\text { Durée unitaire d'ingestion } \\
\quad\left(\mathrm{mn} / \mathrm{g} / \mathrm{kg} \mathrm{P}^{(1,55)} \ldots \ldots \ldots \ldots \ldots \ldots\right. \\
\text { Unitary eating time }\left(\mathrm{mn} / \mathrm{g} / \mathrm{kg} W^{o, i j}\right)\end{array}$ & $4,75 \pm 0,90$ & $4,65 \pm 0,72$ & NS \\
\hline $\begin{array}{l}\text { Durée journalière de rumination }(\mathrm{mn}) \\
\text { Daily time spent on rumination }\end{array}$ & $475 \pm 64$ & $486 \pm 49$ & NS \\
\hline 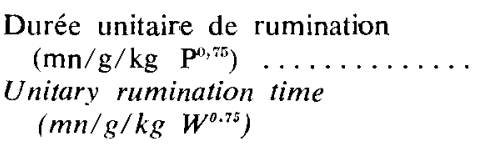 & $7,33 \pm 2,14$ & $5,60 \pm 1,26$ & $\mathrm{P}<0,10$ \\
\hline $\begin{array}{c}\text { Grands repas } \\
\text { suivant la distribution }(\mathrm{n}=2) \\
\text { Main meals following distribution }\end{array}$ & & & \\
\hline $\begin{array}{l}\left.\text { Quantités de MS ingérées ( } \mathrm{g} / \mathrm{kg} \mathrm{P}^{0,75}\right) \\
\text { DM intake }\left(g / \mathrm{kg} W^{0.75}\right)\end{array}$ & $20,9 \pm 5,4$ & $21,8 \pm 6,9$ & NS \\
\hline $\begin{array}{l}\text { Durée d'ingestion }(\mathrm{mn}) \ldots \ldots \ldots \\
\text { Time spent eating }\end{array}$ & $82,6 \pm 18,3$ & $81,6 \pm 23,2$ & NS \\
\hline $\begin{array}{l}\text { Durée unitaire d'ingestion } \\
\quad\left(\mathrm{mn} / \mathrm{g} / \mathrm{kg} \mathrm{P}^{0,75}\right) \ldots \ldots \ldots \ldots \ldots \ldots \\
\text { Unitary eating time }\left(\mathrm{mn} / \mathrm{g} / \mathrm{kg} W^{0.75}\right)\end{array}$ & $4,06 \pm 0,74$ & $3,82 \pm 0,61$ & NS \\
\hline Petits repas - Small meals & & & \\
\hline $\begin{array}{l}\text { Nombre par jour } \ldots \ldots \ldots \ldots \ldots \\
\text { Number per day }\end{array}$ & $6,0 \pm 1,1$ & $7,1 \pm 1,4$ & NS \\
\hline $\begin{array}{l}\text { Quantités de MS ingérées }\left(\mathrm{g} / \mathrm{kg} \mathrm{P}^{0,75}\right) \\
D M \text { intake }\left(g / \mathrm{kg} \mathrm{W}^{0.75}\right)\end{array}$ & $4,4 \pm 1,4$ & $6,5 \pm 2,1$ & $\mathbf{P}<0,10$ \\
\hline $\begin{array}{l}\text { Durée d'ingestion }(\mathrm{mn}) \ldots \ldots \ldots \\
\text { Time spent eating }\end{array}$ & $25,3 \pm 3,5$ & $35,4 \pm 7,1$ & $P<0,10$ \\
\hline $\begin{array}{l}\text { Durée unitaire d'ingestion } \\
\quad\left(\mathrm{mn} / \mathrm{g} / \mathrm{kg} \mathrm{P}^{0, \overline{5}}\right) \ldots \ldots \ldots \ldots \ldots \ldots \\
\text { Unitary eating time }\left(\mathrm{mn} / \mathrm{g} / \mathrm{kg} \mathrm{W}^{0.25}\right)\end{array}$ & $6,18 \pm 1,60$ & $5,67 \pm 1,12$ & NS \\
\hline
\end{tabular}


Ces fourrages ont été fauchés chaque matin, hachés dans un hache-paille (brins de $2-3 \mathrm{~cm})$ et distribués à volonté à liauge (10 p. 100 de refus) en deux repas par jour, à 8 heures et à 16 heures, simultanément à 6 moutons castrés de 2-3 ans (race Texel) et à 5 génisses de 1 an (races Frisonne et Montbéliarde). La période de mesure des quantités ingérées, avec enregistrement du comportement alimentaire selon la technique de Ruckebusch (1963) a été de 6 jours, précédés d'une période d'adaptation de 10 à 14 jours. On appelle «grand repas » celui qui suit la distribution du fourrage, par opposition aux «petits repas», indépendants de la prise alimentaire.

\section{Résultats}

\section{A. Comparaison entre le comportement des moutons et celui des génisses}

La quantité journalière de matière sèche ingérée, rapportée au poids métabolique des animaux, a été plus importante chez les génisses que chez les moutons. La différence s'explique par le nombre légèrement plus élevé de petits repas et, surtout, par leur durée plus longue chez les génisses. La durée des grands repas et la quantité ingérée à cette occasion ont été, en effet, identiques pour les deux espèces. Les génisses ont donc passé plus de temps à ingérer que les moutons (en moyenne 410 contre $314 \mathrm{mn}$ par jour). Comme la durée journalière de rumination est identique, de l'ordre de $480 \mathrm{mn}$, la durée unitaire de rumination est plus faible chez les génisses (tabl. 2).

Les relations statistiques entre les paramètres du comportement des moutons et ceux des génisses sont étroites, sauf pour la durée journalière d'ingestion et le nombre de repas par jour (tabl. 3).

\section{B. Influence de la composition chimique des fourrages sur le comportement des animaux}

Le comportement des moutons et des génisses est en relation avec la qualité des fourrages ingérés. Nous avons fait figurer les coefficients de corrélation des régressions linéaires correspondantes dans le tableau 4. Ainsi, lorsque la teneur en matières azotées et la digestibilité ont augmenté et que la teneur en cellulose brute a baissé, les quantités journalières ingérées ont augmenté, alors que la durée d'ingestion a peu varié et que la durée de rumination a diminué. De même, les durées unitaires d'ingestion, de rumination et de mastication ont diminué. La durée journalière de mastication a alors légèrement diminué. Nous l'avons reportée dans la figure 1, en fonction de la digestibilité des fourrages.

Nous n'avons pas noté d'influence de la qualité des fourrages sur leurs quantités ingérées pendant les grands repas. Par contre, cette influence a été nette sur les quantités ingérées lors de chaque petit repas. A l'inverse, la durée d'ingestion lors des grands repas a diminué avec la qualité des fourrages, alors que celle des petits repas n'a pas été influencée. Les durées unitaires d'ingestion lors des grands repas et des petits repas a toujours varié en sens inverse de la qualité des fourrages. 


\section{Tableau 3}

Relations entre les paramètres du comportement des moutons et ceux des génisses.

Relationship between the behavioural parameters of sheep and those of heifers.

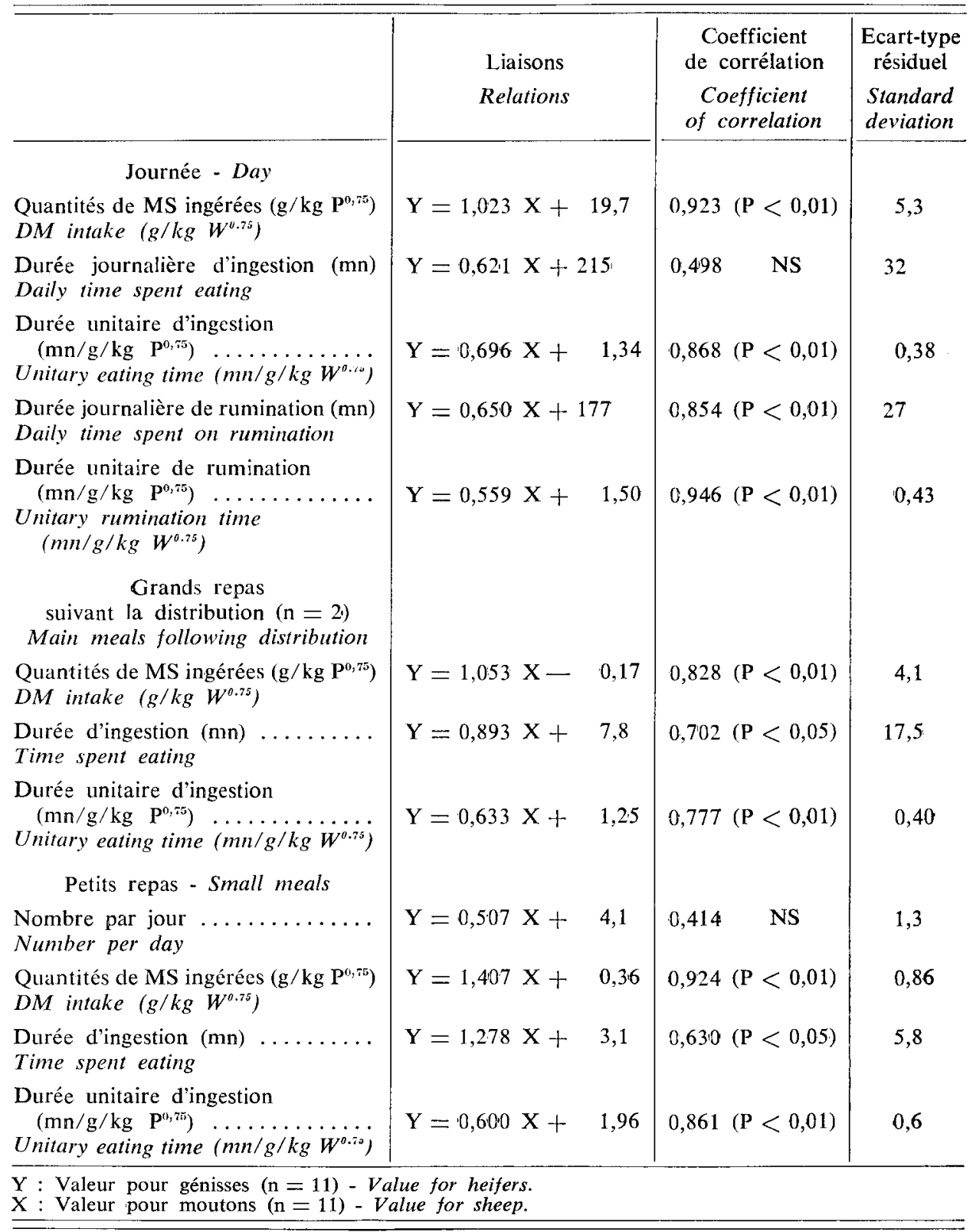




\section{TABLeaU 4}

Influence de la composition chimique sur les paramètres du comportement des animaux (coefficients de corrélation significatif pour $P<0,05$ ).

Influence of chemical composition on the behavioural parameters of the animals (only the significant coefficients of correlation).

\begin{tabular}{|c|c|c|c|c|c|c|}
\hline & \multicolumn{2}{|c|}{$\begin{array}{c}\text { Matières azotées } \\
\text { totales } \\
\text { Crude protein }\end{array}$} & \multicolumn{2}{|c|}{$\begin{array}{l}\text { Cellulose brute } \\
\text { Crude fibre }\end{array}$} & \multicolumn{2}{|c|}{$\begin{array}{l}\text { Digestibilité } \\
\text { de la matière } \\
\text { organique } \\
\text { Organic matter } \\
\text { digestibility }\end{array}$} \\
\hline & $\begin{array}{c}\text { Moutons } \\
\text { Sheep }\end{array}$ & $\begin{array}{c}\text { Génisses } \\
\text { Heifers }\end{array}$ & $\begin{array}{l}\text { Moutons } \\
\text { Sheep }\end{array}$ & $\begin{array}{c}\text { Génisses } \\
\text { Heifers }\end{array}$ & $\begin{array}{c}\text { Moutons } \\
\text { Sheep }\end{array}$ & $\begin{array}{c}\text { Génisses } \\
\text { Heifers }\end{array}$ \\
\hline Journée - Day & & & & & & \\
\hline $\begin{array}{l}\text { Quantités ingérées de MS } \\
\text { DM intake }\end{array}$ & $+0,657$ & $+0,655$ & $\longrightarrow 0,598$ & $-0,530$ & - & 一 \\
\hline $\begin{array}{l}\text { Durée d'ingestion } \ldots . . . \cdots \\
\text { Daily time spent eating }\end{array}$ & - & - & - & - & — 0,594 & 一 \\
\hline $\begin{array}{l}\text { Durée unitaire d'ingestion } \\
\text { Unitary eating time }\end{array}$ & 一 & - & $+0,744$ & $+0,693$ & $-0,710$ & $-0,641$ \\
\hline $\begin{array}{l}\text { Durée de rumination } . . . \\
\text { Daily time spent on rumi- } \\
\text { nation }\end{array}$ & $-0,514$ & $-0,607$ & $+0,524$ & $+0,613$ & $-0,507$ & 一 0,688 \\
\hline $\begin{array}{l}\text { Durée unitaire de rumina- } \\
\quad \text { tion } \ldots \ldots \ldots \ldots \ldots \ldots \\
\text { Unitary time spent rumina- } \\
\quad \text { tion }\end{array}$ & $-0,610$ & $-0,648$ & $+0,665$ & $+0,645$ & $-0,541$ & $-0,555$ \\
\hline $\begin{array}{l}\text { Durée unitaire de mastica- } \\
\text { tion } \ldots \ldots \ldots \ldots \ldots \ldots \\
\text { Unitary time spent chewing }\end{array}$ & $-0,606$ & $-0,580$ & $+0,700$ & $+0,686$ & $-0,600$ & 一 0,607 \\
\hline $\begin{array}{l}\text { Grands nepas } \\
\text { Main meals }\end{array}$ & & & & & & \\
\hline $\begin{array}{l}\text { Quantités ingérées de MS } \\
\text { DM intake }\end{array}$ & - & 一 & - & - & & 一 \\
\hline $\begin{array}{l}\text { Durée d'ingestion ........ } \\
\text { Time spent eating }\end{array}$ & 一 & 一 & $+0,495$ & $+0,569$ & $-0,708$ & $-0,820$ \\
\hline $\begin{array}{l}\text { Durée unitaire d'ingestion } \\
\text { Unitary eating time }\end{array}$ & $-0,606$ & $-0,515$ & $+0,666$ & 一 & $-0,503$ & $-0,527$ \\
\hline $\begin{array}{l}\text { Petits repas } \\
\text { Small meals }\end{array}$ & & & & & & \\
\hline $\begin{array}{l}\text { Nombre par jour } \ldots \ldots \ldots \\
\text { Number per day }\end{array}$ & 一 & - & - & 一 & & 一 \\
\hline $\begin{array}{l}\text { Quantités ingérées de MS } \\
\text { DM intake }\end{array}$ & 一 & - & $\longrightarrow 0,682$ & $-0,597$ & $+0,796$ & $+0,744$ \\
\hline $\begin{array}{l}\text { Durée d'ingestion } . . . \cdots \\
\text { Time spent eating }\end{array}$ & 一 & - & 一 & - & 一 & 一 \\
\hline $\begin{array}{l}\text { Durée unitaire d'ingestion . } \\
\text { Unitary eating time }\end{array}$ & 一 & 一 & $+0,762$ & $+0,680$ & $-0,891$ & $-0,855$ \\
\hline
\end{tabular}




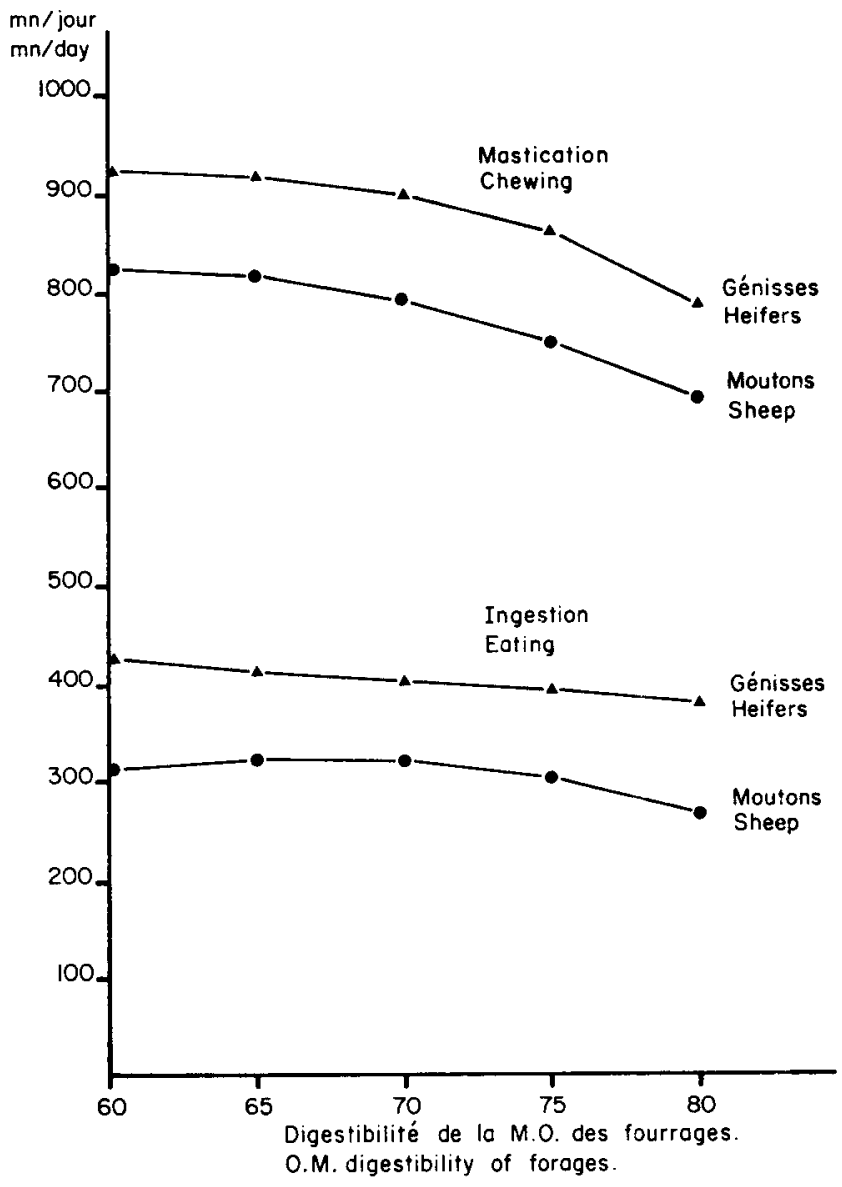

FIG. 1

Evolution des durées journalières d'ingestion et de mastication selon la digestibilité de la matière organique.

Relationship between the times spent eating and chewing and the organic matter digestibility of the forages.

C. Relations entre le comportement et les quantités ingérées

Les relations significatives entre les paramètres du comportement et les quantités ingérées sont rapportées dans le tableau 5. Seules les durées unitaires sont étroitement et négativement corrélées avec les quantités ingérées; les durées d'ingestion et de mastication ont été plutôt indépendantes de ces quantités ingérées, sauf pour la durée de rumination chez les génisses. 


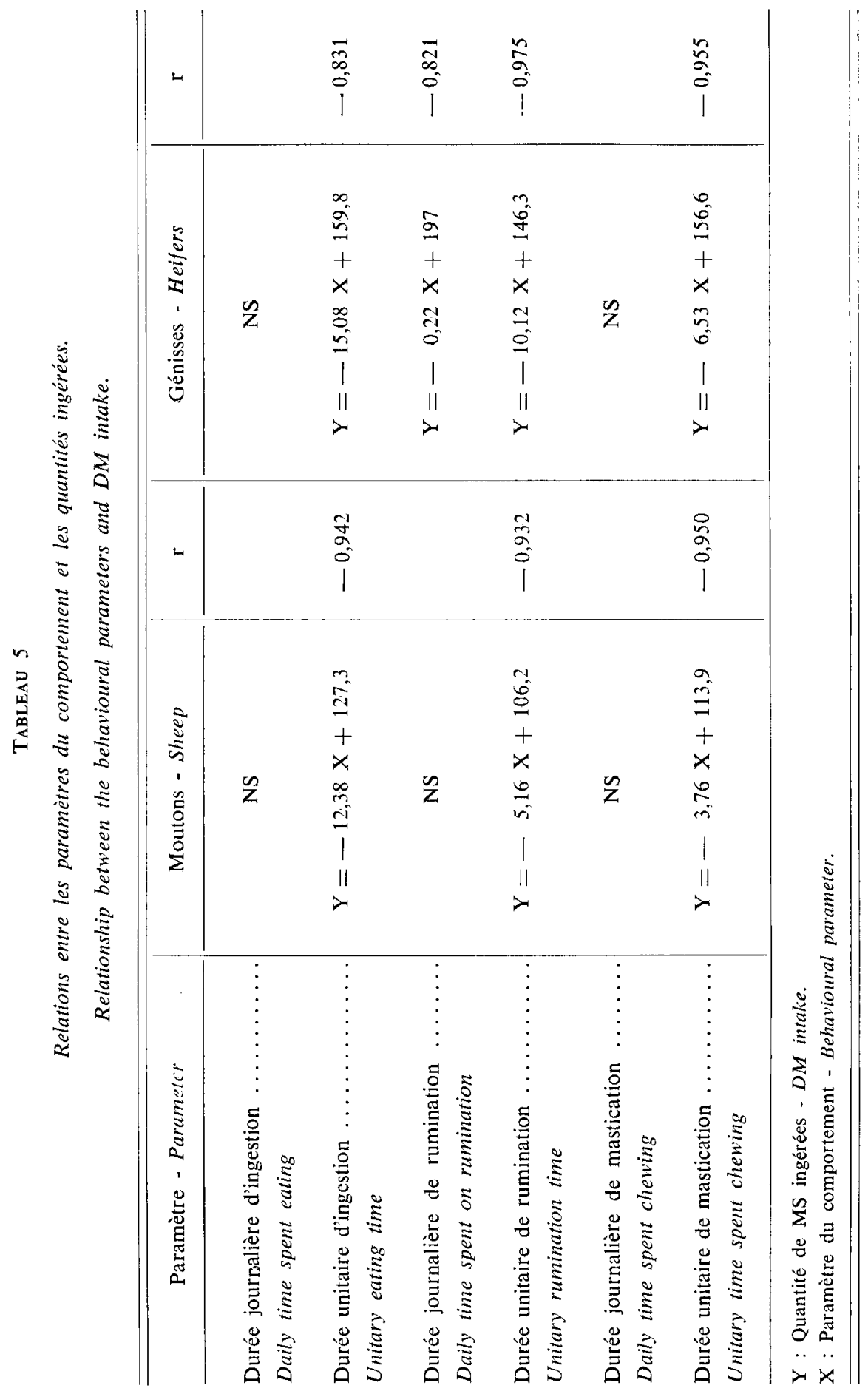




\section{Discussion - conclusion}

Les quantités de fourrages verts ingérées et les paramètres du comportement alimentaire et mérycique des moutons et des génisses varient donc parallèlement et les liaisons calculées sont souvent étroites. Ces résultats peuvent s'expliquer par l'existence d'une régulation de l'appétit de même nature, dans les deux espèces, c'est-à-dire une régulation de type «physique» (BALCH \& CAMPLING, 1962) dont le facteur limitant est l'encombrement du rumen. L'étroitesse de la liaison entre les quantités ingérées par les deux types d'animaux permet aussi d'envisager la prévision assez précise des quantités ingérées par les génisses $\left(\mathrm{QI}_{\mathrm{G}}\right)$ à partir de celles ingérées par les moutons $\left(\mathrm{QI}_{\mathrm{M}}\right)$ :

$$
\mathrm{QI}_{\mathrm{G}}=19,7+1,023 \mathrm{QI}_{\mathrm{MI}}( \pm 5,3 ; \mathrm{r}=0,923)
$$

Demarquilly \& Weiss (1971) avaient, de même, trouvé des liaisons étroites pour les vaches laitières et les bœufs de $500 \mathrm{~kg}$.

Les quantités ingérées (en $\mathrm{g} / \mathrm{kg} \mathbf{P}^{(0,75}$ ) par grands repas sont identiques chez les moutons et les génisses. Les différences de quantités ingérées par jour résultent donc essentiellement du plus grand nombre de petits repas chez les génisses et surtout des quantités plus importantes ingérées à ces moments-là. Cela se traduit par une durée d'ingestion plus longue chez les génisses. Elles ne ruminent cependant pas plus longtemps et passent donc moins de temps à ruminer une même quantité de matière sèche que les moutons, ce qui pourrait expliquer pourquoi les particules de fourrages à la sortie du rumen sont plus grosses chez les génisses (GRENET, 1970).

Quant à l'influence de la composition chimique sur le comportement alimentaire et mérycique, elle est notable, bien que le nombre de mesures soit faible par rapport à nos essais antérieurs (Dulphy \& Demarquilly, 1974). On peut déduire des relations observées que les variations en cellulose brute, en matières azotées, ainsi que la digestibilité, déterminent les durées unitaires d'ingestion et de rumination, ainsi que la durée de rumination. Ce résultat, déjà explicité dans le cas des moutons (Dulphy \& DemarQUILLY, 1974) s'explique par le fait que, lorsque la digestibilité des fourrages augmente (avec baisse de la teneur en cellulose et augmentation de la teneur en matières azotées), leur vitesse de digestion augmente également. Il en résulte une évacuation plus rapide du rumen, d'où l'augmentation du nombre des repas (Dulphy \& BECHET, 1976) ou, comme c'est le cas dans cet essai, des quantités ingérées par petits repas.

On constate, en outre, que l'influence de la teneur en cellulose brute sur la durée unitaire de rumination est beaucoup plus faible chez les génisses que chez les moutons (coefficients de régression respectivement de $+0,17$ et $+0,30$ ). Cela provient du fait que la mastication mérycique n'a pas besoin d'être aussi efficace pour les génisses, comme le montrent les relations du tableau 5. En effet, pour une même variation de la durée unitaire de rumination, les quantités ingérées varient deux fois plus chez les génisses que chez les moutons.

En définitive, les paramètres du comportement alimentaire des génisses sont comparables à ceux des moutons. Lutilisation de l'espèce ovine étant relativement plus aisée que celle de l'espèce bovine, il était donc intéressant de savoir que, moyennant le recours à certaines relations, l'extrapolation aux génisses des résultats obtenus sur des moutons est très satisfaisante. 


\section{Summary}

\section{Feeding behaviour and rumination in sheep and heifers fed green forages}

Eleven fresh forages (tabl. 1) were given simultaneously to sheep and heifers to compare their feeding and rumination behaviours.

Heifers ingested more $\left(89.7 \mathrm{vs} 68.4 \mathrm{~g} \mathrm{DM} / \mathrm{kg} \mathrm{W}^{0.75}\right)$ and during a longer time (410 vs $314 \mathrm{mn} /$ day) than sheep. They ruminated more rapidly a same DM quantity (5.60 vs $7.33 \mathrm{mn} / \mathrm{g} / \mathrm{kg} \mathrm{W}^{0.55}$ ). Sheep and heifers ingested similar amounts at the same rate during the «main meals» following immediately the two distributions of forages. Moreover, heifers made more meals than sheep and, above all, ingested more during the "small meals 》 (6.5 vs $4.4 \mathrm{~g} \mathrm{DM} / \mathrm{kg} \mathrm{W}^{0.55}$ ) (cf. tabl. 2).

The different parameters of the feeding and rumination behaviours of heifers and sheep were closely correlated, except the daily time spent eating and the number of meals per day (tabl. 3).

The influence of the forage chemical composition on the feeding and rumination behaviours (tabl. 4) was also studied. When the quality of the forage increased, the daily intake also increased, but the feed intake during the «main meals» was constant. In the same conditions the time spent eating was almost constant for each forage, but the daily time spent on rumination decreased and the daily time spent chewing also decreased (fig. 1).

Finally, for each animal species the relations between feeding and rumination behaviours and intake were examined (tabl. 5).

All these results are discussed in connection with the concept of physical control of feed intake.

\section{Références bibliographiques}

Balch C.C., Campling R.C., 1962. Regulation of voluntary feed intake in ruminants. Nutr. Abstr. Rev., 32, 669-686.

Demarquilly C., Weiss Ph., 1971. Liaison entre les quantités de matière sèche de fourrages verts ingérées par les moutons et celles ingérées par les bovins. Ann. Zootech., 20, 119-134.

Dulphy J.P., Bechet G., 1976. Influence du stade de végétation et de l'espèce végétale sur le comportement alimentaire et mérycique de moutons recevant des fourrages verts hachés. Ann. Zootech., 25, 505-519.

DulPhy J.P., Demarquilly C., 1974. Etude du comportement alimentaire et mérycique des moutons recevant des fourrages verts hachés. Ann. Zootech., 23, 193-212.

Dulphy J.P., Michalet-Doreau B., Demarquilly C., 1984. Etude comparée du comportement alimentaire et mérycique d'ovins et de bovins recevant des fourrages verts et des ensilages. Ann. Zootech. (en préparation).

Dulphy J.P., Remond B., Theriez M., 1979. Ingestive behaviour and related activities in ruminants. Proc. 5th Int. Symposium on digestive physiology and metabolism in ruminants, Clermont-Ferrand, 3-7 septembre. Y. Ruckebusch and P. Thivend Eds. M.T.P. Press Ltd., Lancaster, pp. 103-122.

Grenet E., 1970. Taille et structure des particules végétales au niveau du feuillet et des fèces chez les bovins. Ann. Biol. anim. Biochim. Biophys., 10, 643-657.

RuckeBusch Y., 1963. Recherches sur la régulation centrale du comportement alimentaire chez les ruminants. Thèse Doc. Sci. nat., Univ. de Lyon, 213 pages. 